\title{
How polymer additives reduce the pour point of hydrocarbon solvents containing wax crystals
}

\author{
Bernard P. Binks, Paul D.I. Fletcher* and Noel A. Roberts \\ Surfactant \& Colloid Group, Department of Chemistry, \\ University of Hull, Hull. HU6 7RX. U.K.
}

John Dunkerley, Hannah Greenfield, Antonio Mastrangelo and Kieran Trickett Lubrizol Limited, The Knowle, Nether Lane, Hazelwood, Derby, Derbyshire DE56 4AN

\section{Electronic Supplementary Material}

\section{PPD Polymers}

ESI Table 1. The four maleic anhydride (M)-styrene (S)-methyl methacrylate (A) copolymers have the general structure shown below in which $\mathrm{R}$ is a linear chain alkyl group. The polymers contain two $\mathrm{R}$ groups per maleic anhydride monomer unit plus one $\mathrm{R}$ group per methyl methacrylate monomer unit (when present). The number $\left(\mathrm{M}_{\mathrm{n}}\right)$ and weight $\left(\mathrm{M}_{\mathrm{w}}\right)$ average molar masses were estimated using gel permeation chromatography. The maleic anhydridestyrene copolymers are alternating in structure. The methyl methacrylate distribution in the maleic anhydride-styrene-methyl methacrylate terpolymer is thought to be approximately random within the (mainly) alternating structure of the parent maleic anhydride-styrene copolymer.

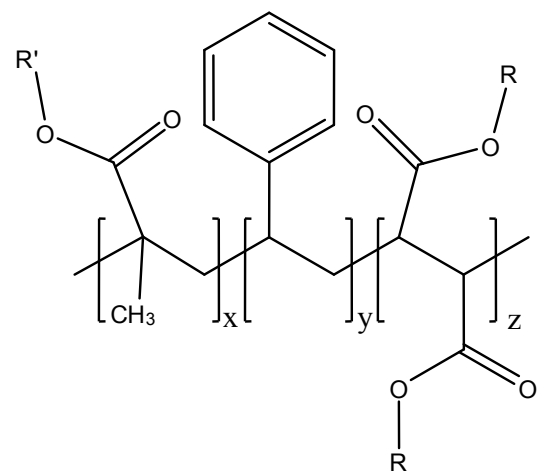

\begin{tabular}{|c|c|c|c|c|}
\hline PPD polymer & $\begin{array}{c}\text { Monomer mole } \\
\text { ratio } \\
\text { M:S:A }\end{array}$ & $\mathbf{M}_{\mathbf{n}} / \mathbf{k D a}$ & $\begin{array}{c}\text { Polydispersity } \\
\text { ratio } \mathbf{M}_{\mathbf{w}} / \mathbf{M}_{\mathbf{n}}\end{array}$ & $\begin{array}{c}\text { Chain length } \\
\text { of R side } \\
\text { chain }\end{array}$ \\
\hline HMn-MS-C18-22 & $1: 1: 0$ & 69 & 5.9 & $18-22$ \\
\hline LMn-MS-C18-22 & $1: 1: 0$ & 34 & 1.7 & $18-22$ \\
\hline LMn-MSA-C18-22 & $1: 1: 0.05$ & 27 & 1.9 & $18-22$ \\
\hline LMn-MSA-C12 & $1: 1: 0.05$ & 20 & 2.0 & 12 \\
\hline
\end{tabular}




\section{Temperature hysteresis of the wax solubility boundary}

For a wax solution, the temperature at which wax crystals are visible on cooling the solution $\left(\mathrm{T}_{\text {cool }}\right)$ is generally observed to be a few degrees below the temperature at which the last wax crystals are observed to vanish on heating the solution $\left(T_{\text {heat }}\right)$. The following experiment was performed in order to assess which of the measured values of $\mathrm{T}_{\text {cool }}$ or $\mathrm{T}_{\text {heat }}$ corresponds most closely to the equilibrium solubility boundary. The values of $\mathrm{T}_{\text {cool }}=19.1{ }^{\circ} \mathrm{C}$ and $\mathrm{T}_{\text {heat }}=21.6$ ${ }^{\circ} \mathrm{C}$ for a $15 \mathrm{wt} \%$ solution of $\mathrm{C} 24$ in heptane were measured using a rate of temperature change of $0.1{ }^{\circ} \mathrm{C}$ per minute and are indicated in ESI Figure 1 as the dashed vertical lines. Next, the fully-dissolved solution was cooled from slightly above $\mathrm{T}_{\text {heat }}$ to different temperatures below $T_{\text {heat }}$ but above $\mathrm{T}_{\text {cool }}$. The visual appearance was monitored and the time at which crystals first appeared was recorded. ESI Figure 1 indicates the temperature and whether crystals were visible (filled circles) or not (unfilled circles) over the incubation periods. The time for crystals to first appear decreased exponentially as the incubation temperature was decreased below $T_{\text {heat }}$. The conclusion drawn from this experiment is that $\mathrm{T}_{\text {heat }}$ corresponds closely to the equilibrium temperature of the solubility boundary. This result is expected since the process of crystal formation (at $\mathrm{T}_{\text {cool }}$ ) is predicted to be kinetically slow since crystal nucleation requires an energy barrier to be overcome. The process of crystal melting and dissolution (at $\mathrm{T}_{\text {heat }}$ ) does not exhibit an energy barrier and hence $\mathrm{T}_{\text {heat }}$ should correspond closely to the equilibrium solubility boundary temperature.

ESI Figure 1. Variation of the time for crystals to first appear with incubation temperature $T$ relative to $\mathrm{T}_{\text {heat }}$ (expressed as $\left(\mathrm{T}-\mathrm{T}_{\text {heat }}\right)$ ) for $15 \mathrm{wt} \% \mathrm{C} 24$ in heptane. $\mathrm{T}_{\text {cool }}$ and $\mathrm{T}_{\text {heat }}$ are shown as the dashed vertical lines. Unfilled circles indicate that no crystals are present and the filled circles indicate crystals are observed. The solid line shows the best fit to the equation indicated.

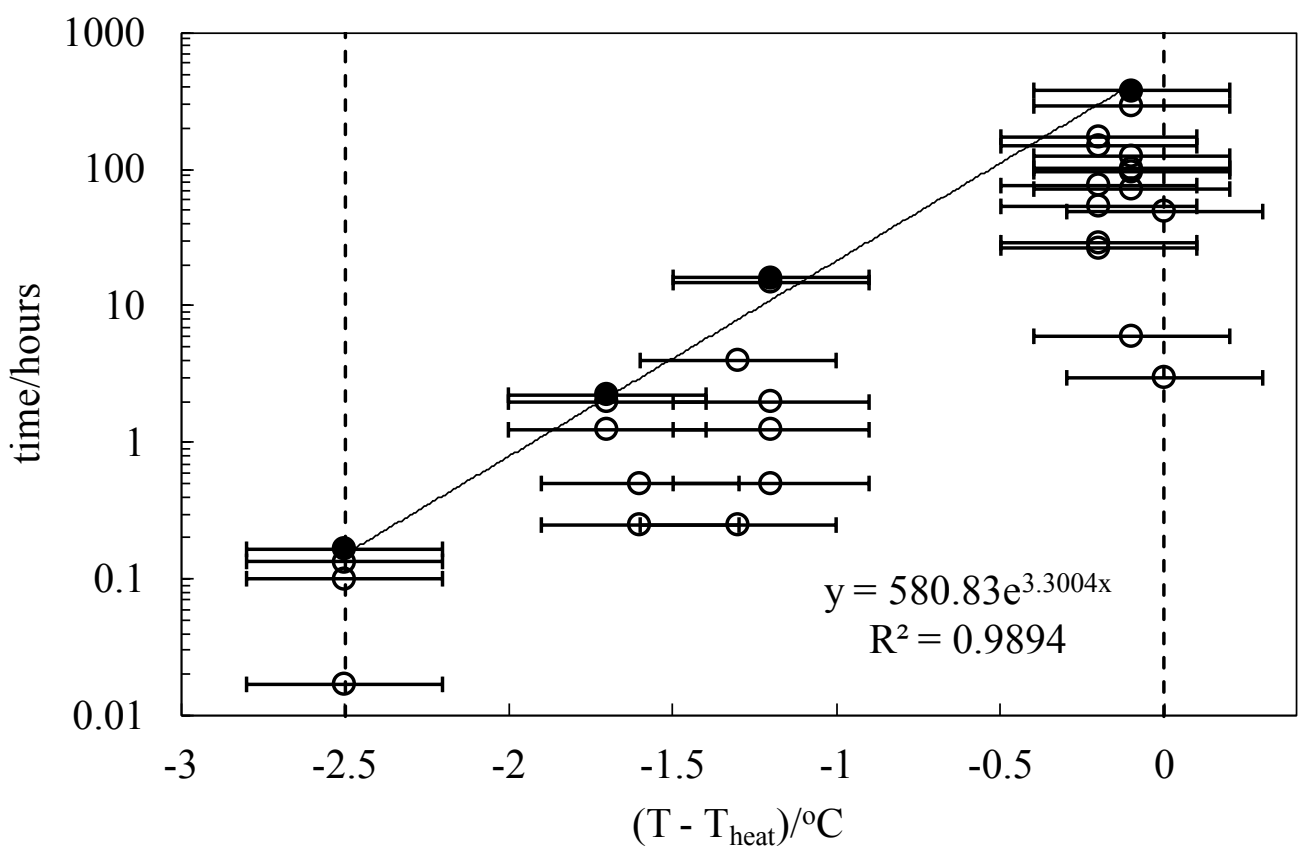


3. Variation of PPD polymer solubility temperature with polymer concentration.

ESI Figure 2. Variation of PPD solubility boundary temperature with polymer concentration in heptane (upper plot) and toluene (lower plot) as solvent. Solubility temperatures for LMn-MSA-C12 were all below $-10{ }^{\circ} \mathrm{C}$ in both solvents and are not shown. The lines are guides for the eye.
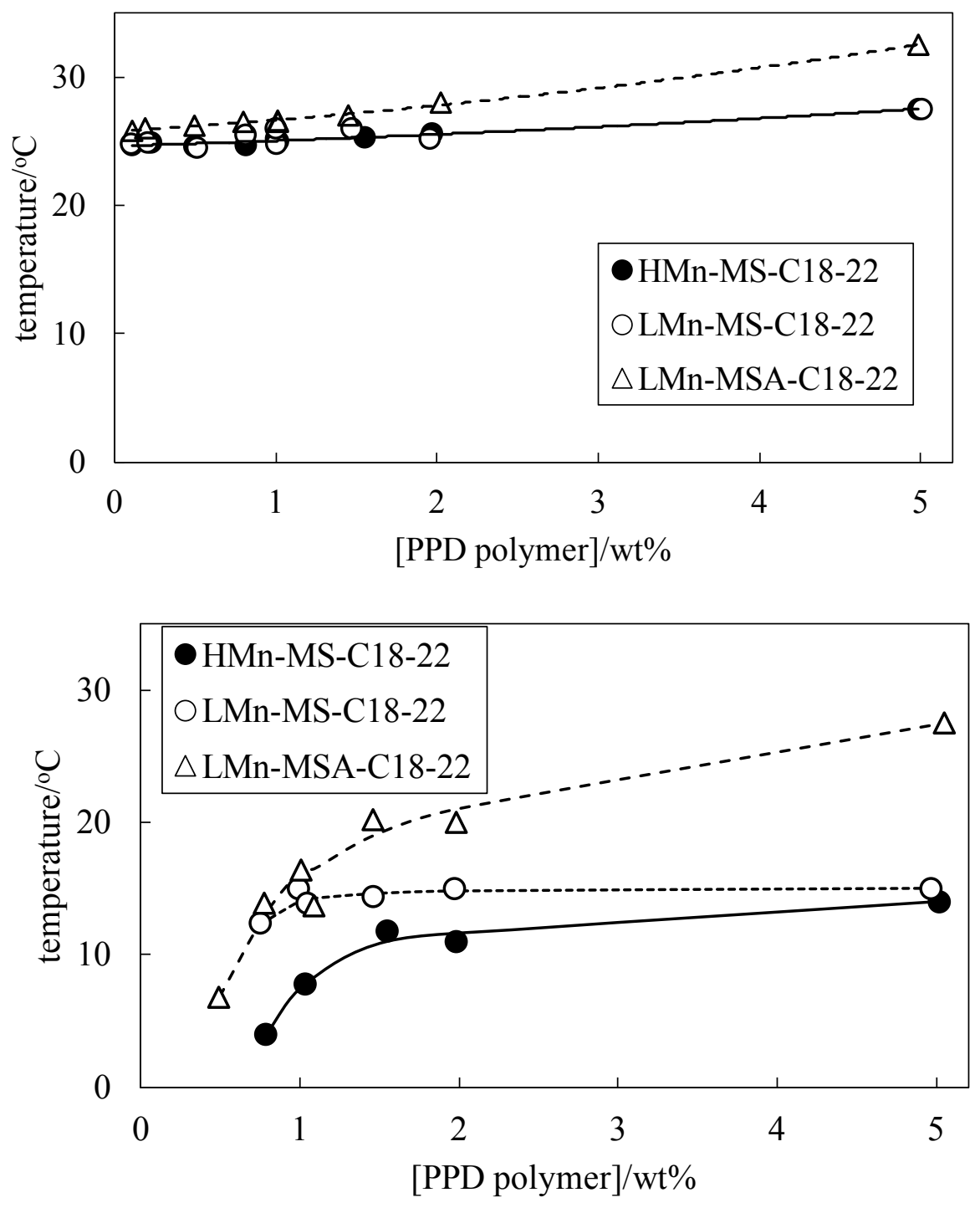


\section{Variation of pour point temperature with PPD polymer concentration.}

ESI Figure 3. Variation of pour point temperature with $\mathrm{C} 24$ wax concentration in toluene for different concentrations of LMn-MSA-C12 (upper plot) and LMn-MSA-C1822 (lower plot). The solid curves show the calculated wax solubility curves for comparison.
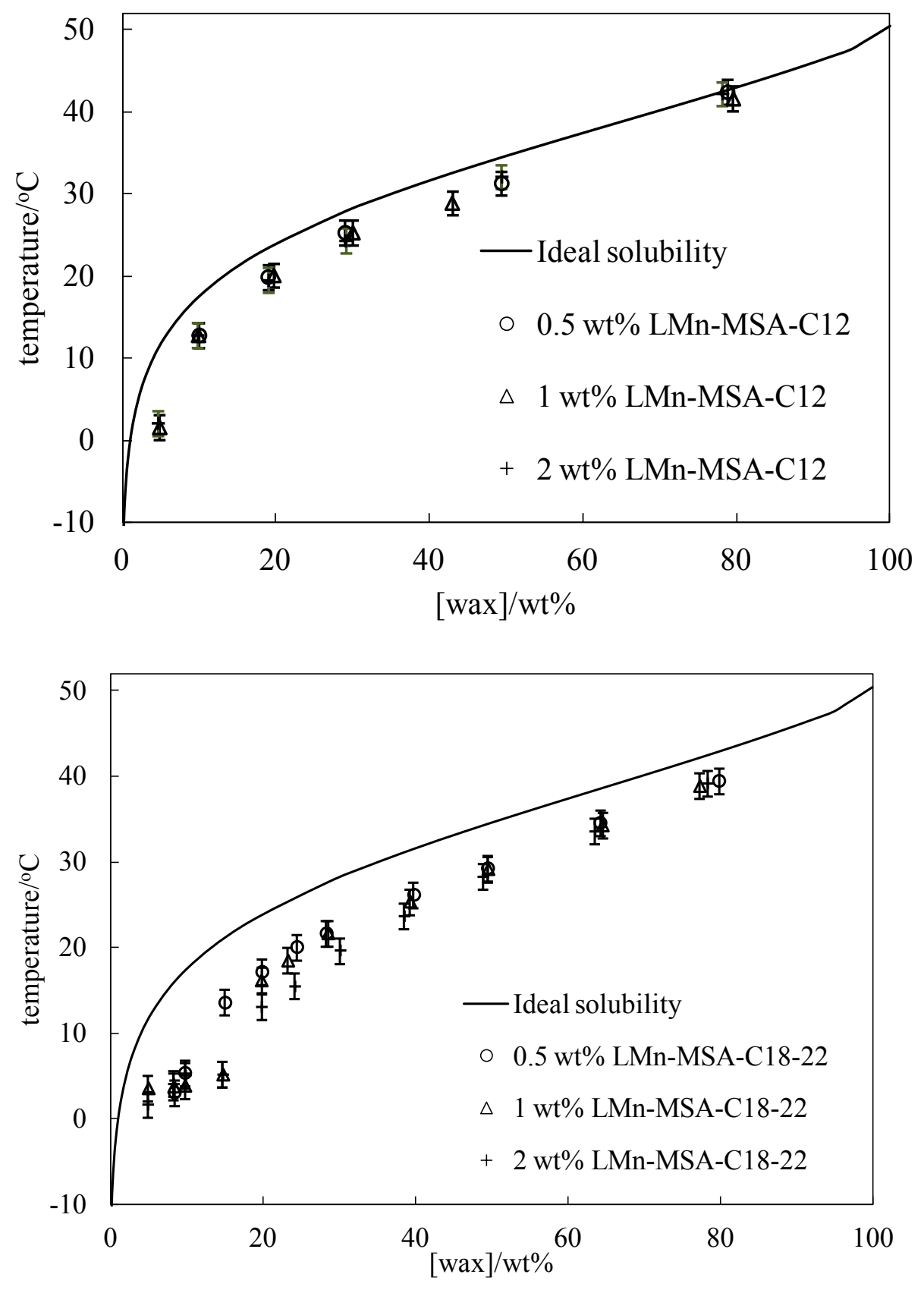


\section{Measurement of axial ratio $(\mathrm{h} / \mathrm{d})$ of precipitated wax crystals.}

ESI Figure 4. Examples of wax crystal SEM images showing the arrowed dimensions of individual crystals used to construct the cumulative distribution plots for $h$ and d.

$20 \mathrm{wt} \% \mathrm{C} 24$ in toluene with no PPD polymer

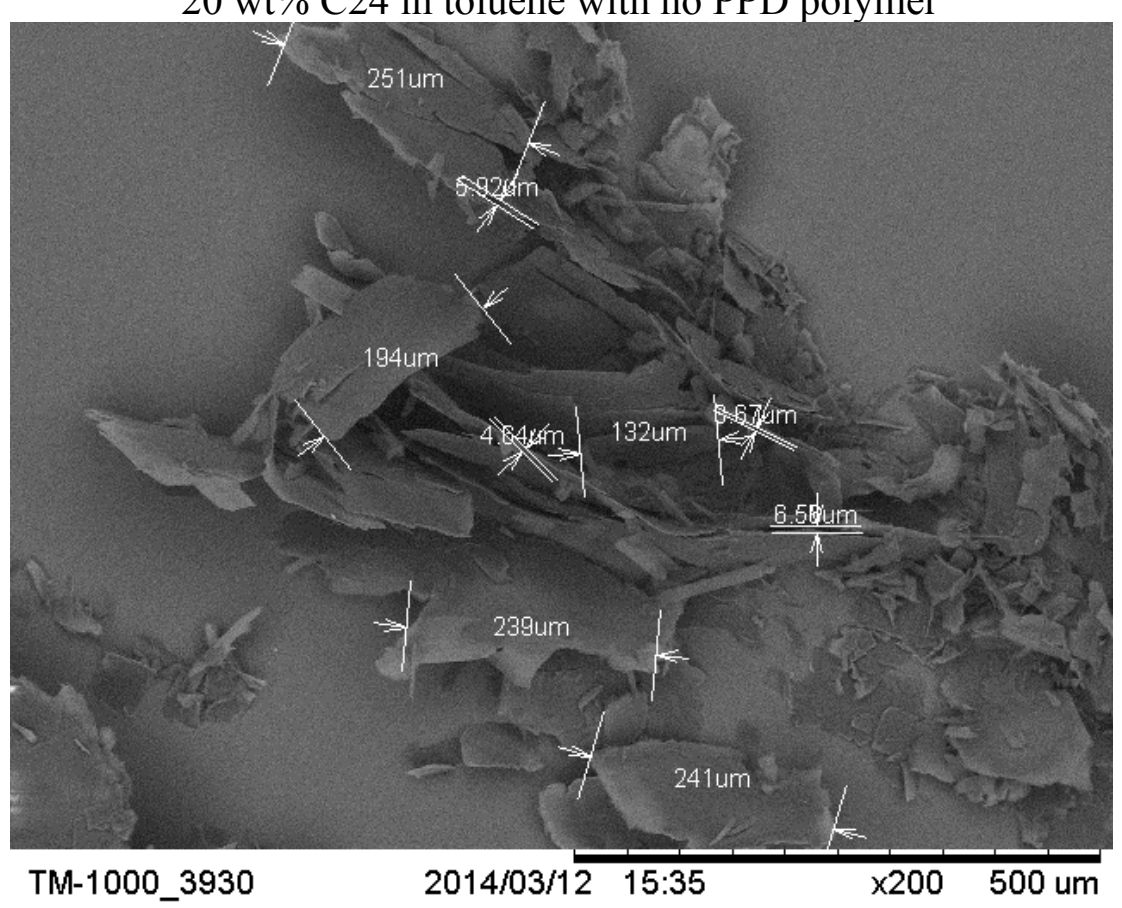

$20 \mathrm{wt} \% \mathrm{C} 24$ in toluene with $1 \mathrm{wt} \%$ LMn-MS-C18-22

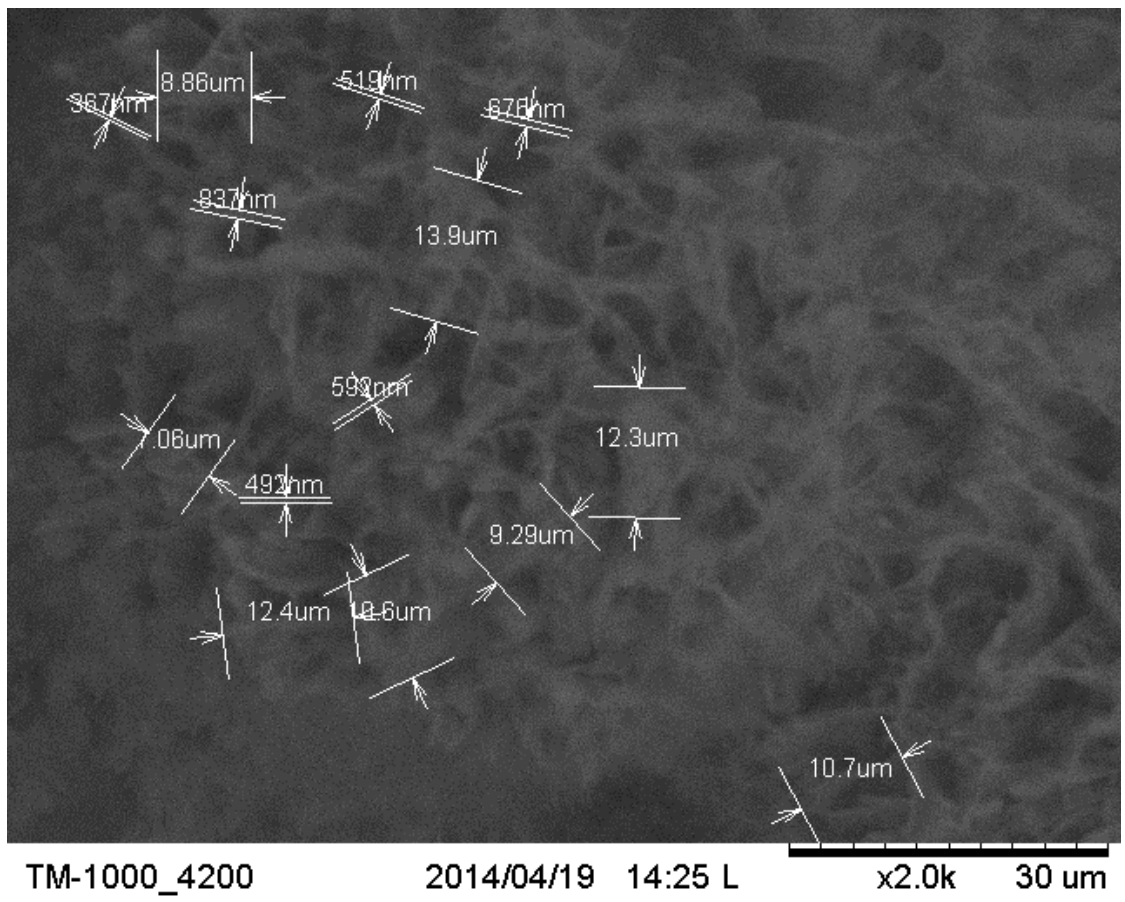


$20 \mathrm{wt} \% \mathrm{C} 24$ in toluene with $1 \mathrm{wt} \%$ LMn-MS-C18-22 (higher magnification)

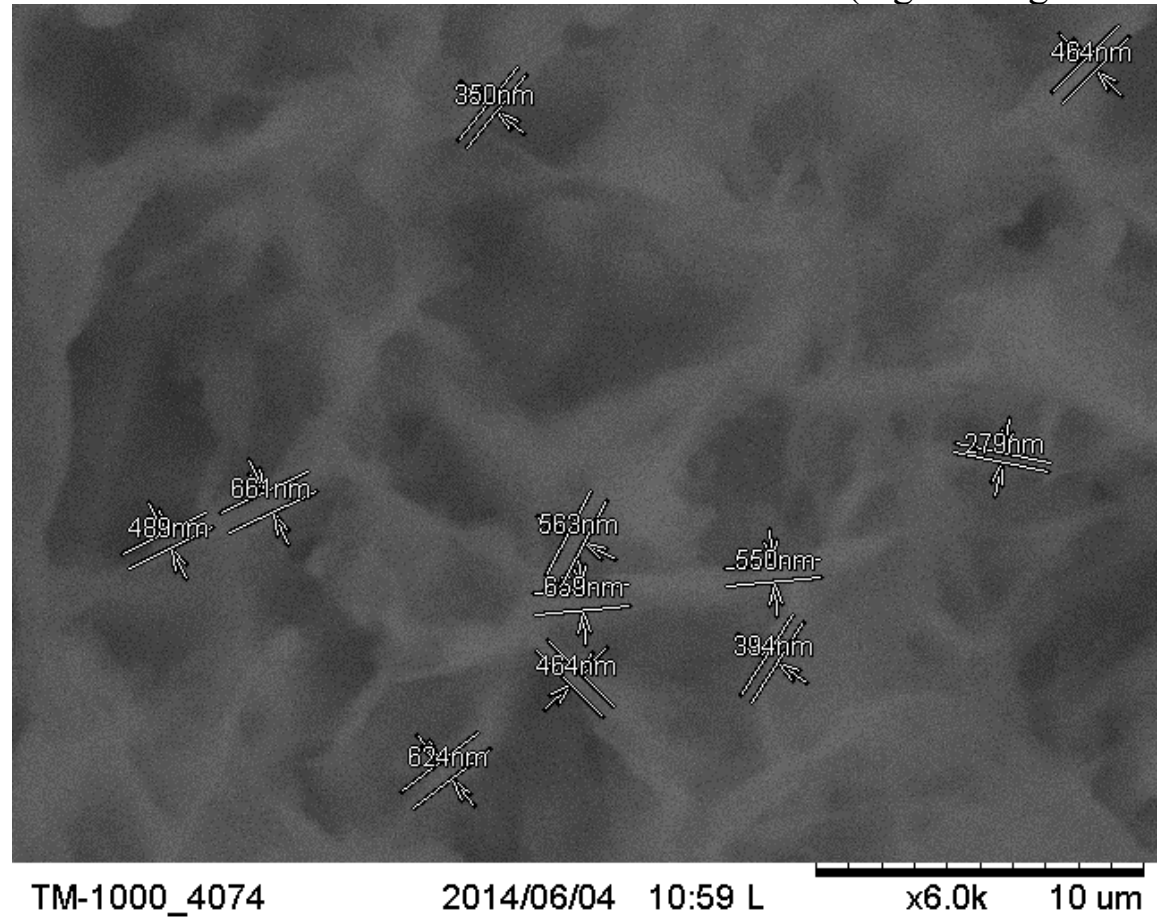

$20 \mathrm{wt} \%$ C36 in heptane with no PPD polymer

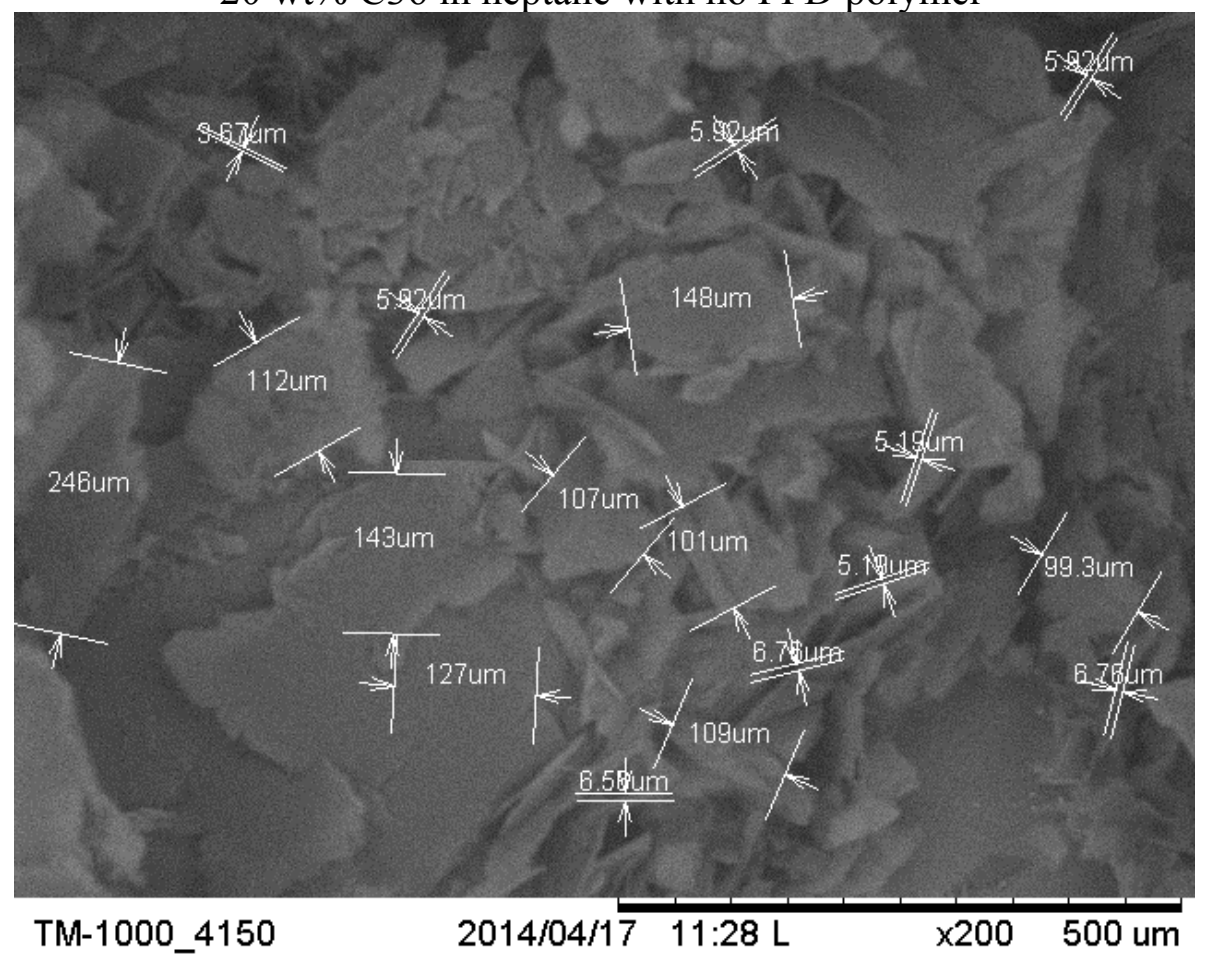


$20 \mathrm{wt} \% \mathrm{C} 36$ in toluene with $1 \mathrm{wt} \%$ LMn-MSA-C12

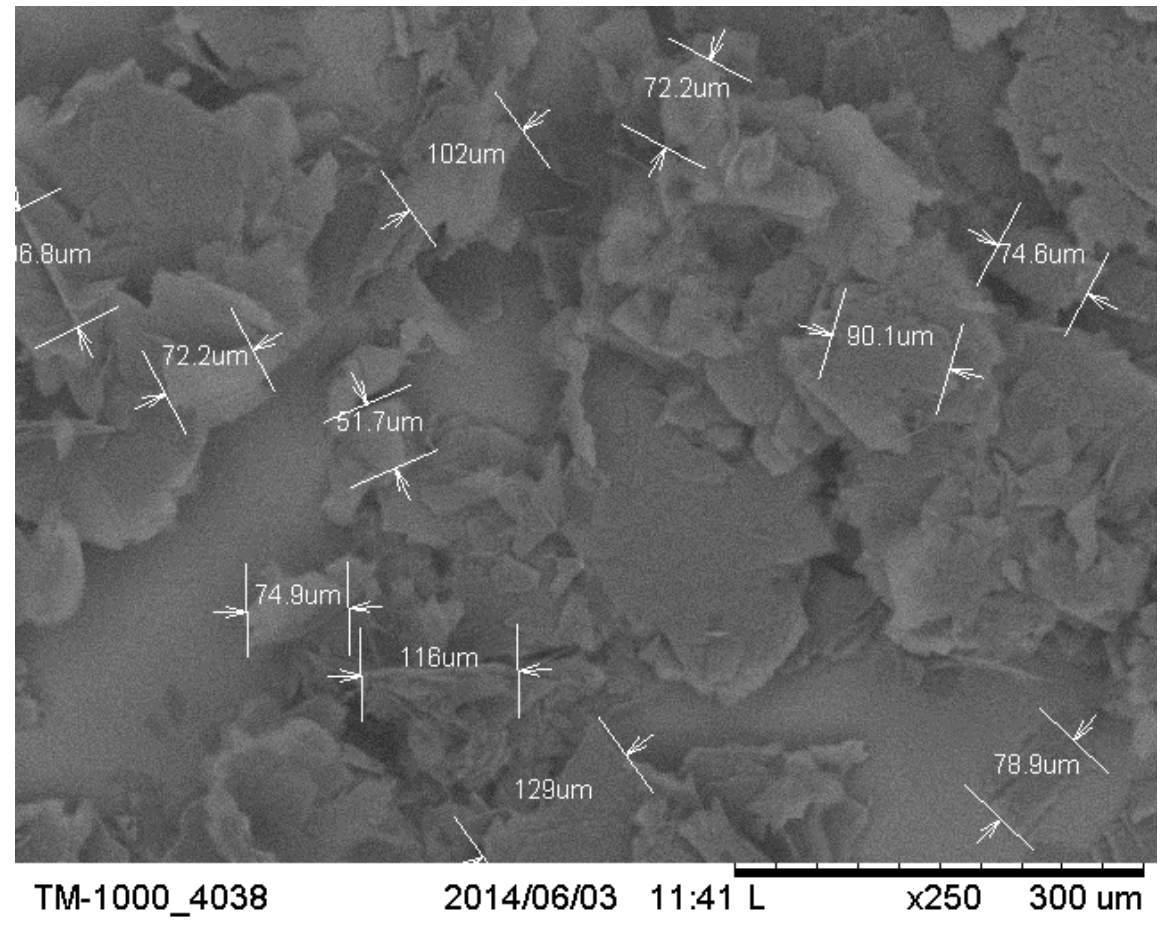

$20 \mathrm{wt} \% \mathrm{C} 36$ in toluene with $1 \mathrm{wt} \%$ LMn-MSA-C12 (higher magnification)

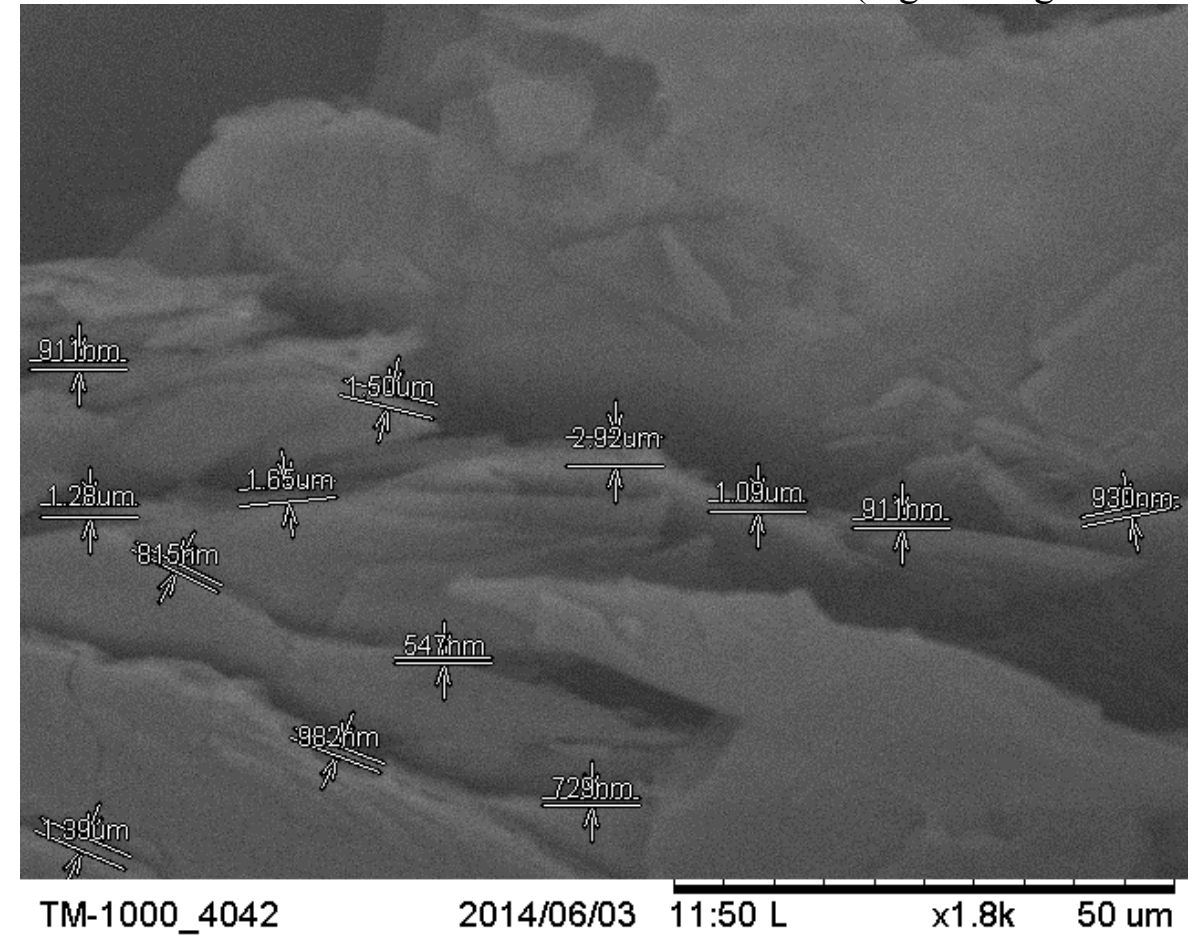


ESI Table 2. Results of repeated experiments to determine the reproducibility/repeatability of $\mathrm{h}$ and $\mathrm{d}$ values at $50 \%$ cumulative frequency from SEM images of precipitated alkane wax crystals. $\mathrm{n} / \mathrm{d}$ indicates the value was not determined.

\begin{tabular}{|c|c|c|c|c|c|c|}
\hline & \multicolumn{2}{|c|}{ Experiment 1 } & \multicolumn{2}{c|}{ Experiment 2 } & \multicolumn{2}{c|}{ Experiment 3 } \\
\hline System & $\mathbf{h} / \boldsymbol{\mu m}$ & $\mathbf{d} / \boldsymbol{\mu m}$ & $\mathbf{h} / \boldsymbol{\mu m}$ & $\mathbf{d} / \boldsymbol{\mu} \mathbf{m}$ & $\mathbf{h} / \boldsymbol{\mu m}$ & $\mathbf{d} / \boldsymbol{\mu m}$ \\
\hline C24 from heptane & $\mathrm{n} / \mathrm{d}$ & 122 & 0.78 & 103 & 0.80 & 109 \\
\hline C24 from toluene & $\mathrm{n} / \mathrm{d}$ & 193 & 1.1 & 202 & 1.0 & 190 \\
\hline C36 from heptane & $\mathrm{n} / \mathrm{d}$ & 115 & 0.56 & 99 & 0.80 & 86 \\
\hline C36 from toluene & $\mathrm{n} / \mathrm{d}$ & 93 & 1.4 & 98 & $\mathrm{n} / \mathrm{d}$ & $\mathrm{n} / \mathrm{d}$ \\
\hline
\end{tabular}


ESI Figure 5. Upper plot: Cumulative distributions of $\mathrm{h}$ and $\mathrm{d}$ for 20 and $40 \mathrm{wt} \% \mathrm{C} 24$ in heptane in the absence of PPD. Lower plot: Comparison of cumulative distributions of $\mathrm{h}$ and $\mathrm{d}$ for $20 \mathrm{wt} \% \mathrm{C} 24$ in heptane using crystal samples extracted following different temperature changes as described in the text. The uncertainties in the individual values of $h$ and $d$ at $50 \%$ cumulative frequency are estimated to be approximately $\pm 40 \%$ and $\pm 30 \%$ respectively; the uncertainties in $\mathrm{h}$ are slightly larger than for $\mathrm{d}$.
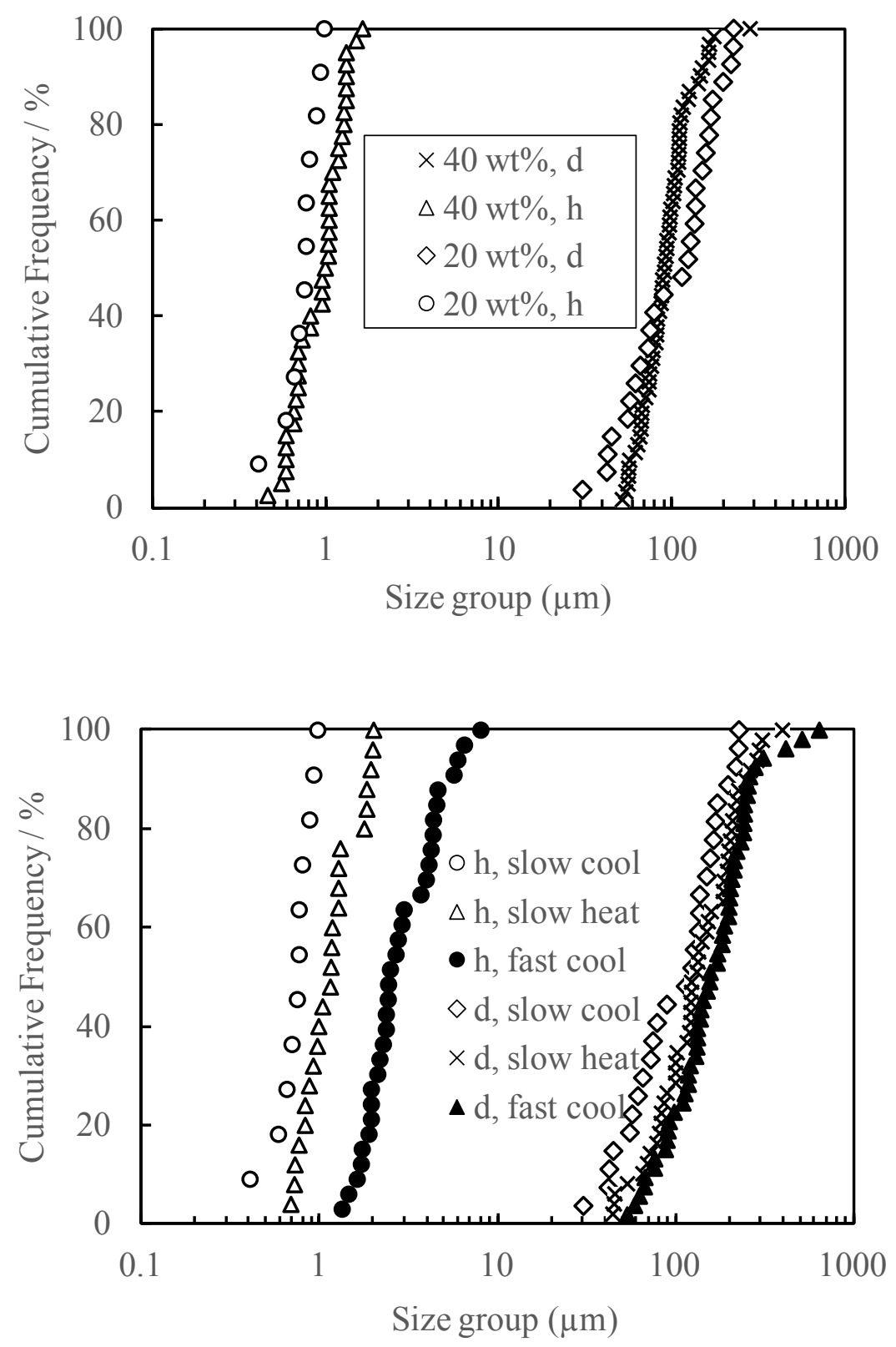
ESI Figure 6. Variation of polymer PPD efficacy (plotted as $\left.\left(\phi^{*}{ }_{1 \mathrm{wt} \% \mathrm{PPD}}-\phi^{*}{ }_{\text {noPPD }}\right)\right)$ versus the difference in polymer PPD and wax solubility temperatures for all wax/solvent/PPD systems with $\mathrm{T}_{\text {heat }}$ for either $50 \mathrm{wt} \%$ wax (upper plot) or 100 wt $\%$ wax (lower plot). The unfilled symbols refer to systems containing LMnMSA-C12 for which the solubility temperature in both solvents was determined only as being less than $-10^{\circ} \mathrm{C}$; for these data points the true position on the relative temperature scale is somewhere below the position plotted (using $-10^{\circ} \mathrm{C}$ as the PPD solubility temperature). The horizontal solid line marks zero pour point depression and the curved dashed line is a guide for the eye.
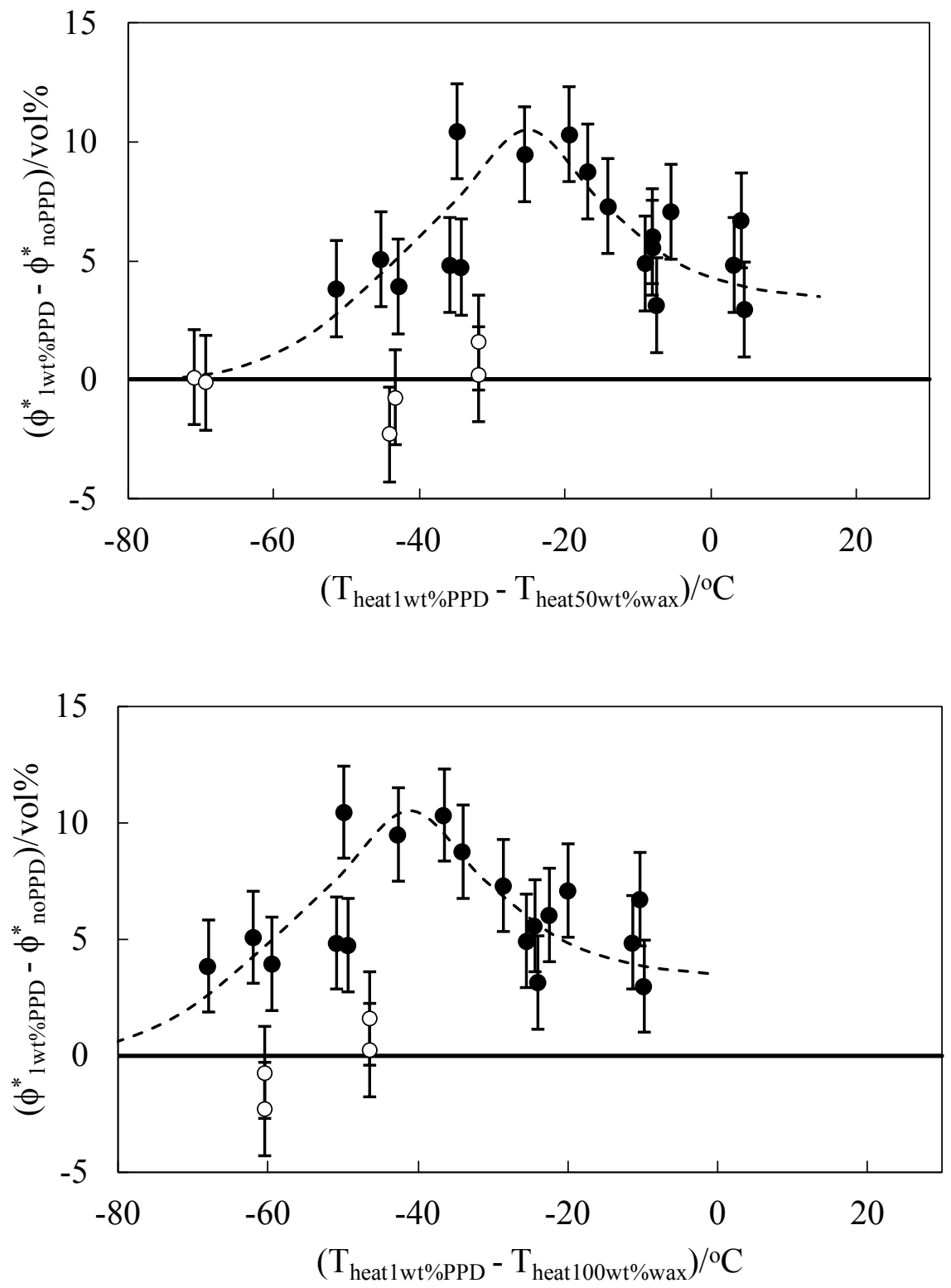